\title{
BMJ Open A randomised controlled pilot trial evaluating feasibility and acceptability of a computer-based tool to identify and reduce harmful and hazardous drinking among adolescents with alcohol-related presentations in Canadian pediatric emergency departments
}

\author{
Amanda S Newton, ${ }^{1,2}$ Nadia Dow, ${ }^{1,3}$ Kathryn Dong, ${ }^{4}$ Eleanor Fitzpatrick, ${ }^{5}$ \\ T Cameron Wild, ${ }^{6}$ David W Johnson, ${ }^{7}$ Samina Ali, ${ }^{1,2}$ Ian Colman, ${ }^{8}$ \\ Rhonda J Rosychuk, ${ }^{1,2}$ Pediatric Emergency Research Canada
}

To cite: Newton AS, Dow N, Dong $\mathrm{K}$, et al. A randomised controlled pilot trial evaluating feasibility and acceptability of a computer-based tool to identify and reduce harmful and hazardous drinking among adolescents with alcoholrelated presentations in Canadian pediatric emergency departments. BMJ Open 2017;7:e015423. doi:10.1136/ bmjopen-2016-015423

- Prepublication history and additional material for this paper are available online. To view these files please visit the journal online (http://dx.doi. org/10.1136/bmjopen-2016015423).

Received 6 December 2016 Revised 13 June 2017 Accepted 5 July 2017

CrossMark

For numbered affiliations see end of article.

Correspondence to

Dr Amanda S Newton;

mandi.newton@ualberta.ca

\section{ABSTRACT}

Objective This study piloted procedures and obtained data on intervention acceptability to determine the feasibility of a definitive randomised controlled trial (RCT) of the effectiveness of a computer-based brief intervention in the emergency department (ED).

Design Two-arm, multi-site, pilot RCT.

Setting and participants Adolescents aged 12-17 years presenting to three Canadian pediatric EDs from July 2010 to January 2013 for an alcohol-related complaint. Interventions Standard medical care plus computerbased screening and personalised assessment feedback (experimental group) or standard care plus computerbased sham (control group). ED and research staff, and adolescents were blinded to allocation.

Outcomes Main: change in alcohol consumption from baseline to 1 - and 3 months post-intervention. Secondary: recruitment and retention rates, intervention acceptability and feasibility, perception of group allocation among ED and research staff, and change in health and social services utilisation.

Results of the 340 adolescents screened, 117 adolescents were eligible and 44 participated in the study (37.6\% recruitment rate). Adolescents allocated to the intervention found it easy, quick and informative, but were divided on the credibility of the feedback provided (agreed it was credible: $44.4 \%$, disagreed: $16.7 \%$, unsure: $16.7 \%$, no response: $22.2 \%$ ). We found no evidence of a statistically significant relationship between which interventions adolescents were allocated to and which interventions staff thought they received. Alcohol consumption, and health and social services data were largely incomplete due to modest study retention rates of $47.7 \%$ and $40.9 \%$ at 1 - and 3 months post-intervention, respectively.

Conclusions A computer-based intervention was acceptable to adolescents and delivery was feasible in the ED in terms of time to use and ease of use. However,
Strengths and limitations of this study

This trial provided important information regarding the feasibility and acceptability of a computerbased intervention for alcohol-related emergency department visits.

- The assessment of intervention credibility was novel to our study.

- Unintended disruption to the randomisation scheme created an imbalance between the control and experimental group allocation.

- The retention rates at 1 - and 3 month follow-up were modest.

adjustments are needed to the intervention to improve its credibility. A definitive RCT will be feasible if protocol adjustments are made to improve recruitment and retention rates; and increase the number of study sites and research staff.

Trial registration clinicaltrials.gov NCT01146665

\section{BACKGROUND}

Alcohol use escalates rapidly during adolescence, ${ }^{1}$ and can have long-lasting adverse effects on adolescent health and well-being. Adolescents who begin drinking before age 15 years are at a four-fold increased risk of developing alcohol dependence compared with those who have their first drink later in adolescence. ${ }^{2-4}$ Alcohol use that is harmful (ie, associated with harms resulting from consumption patterns or contexts of alcohol use) and hazardous (ie, excessive consumption that increases risk for future 
adverse health events) during adolescence predicts later adult substance use disorders ${ }^{2-7}$ and related functioning difficulties such as unemployment. ${ }^{8}$ While many adolescents experiment with alcohol without significant problems, others are at high risk for later abuse and dependence $^{9-11}$ and can experience significant morbidity and mortality. ${ }^{2-412-15}$

The emergency department (ED) is an opportune setting to intervene at a time directly coupled to the consequences of an alcohol-related event or problem. ${ }^{1617}$ Alcohol underlies $1 \%$ of all ED visits by $13-15$ year olds and $2 \%$ of visits by $16-17$ year olds. ${ }^{18}$ Hazardous and harmful alcohol use is also one of the most common reasons adolescents seek emergency mental healthcare. ${ }^{19}$ Because adolescents may not recognise their alcohol consumption as being problematic, know where to seek assistance, or may be embarrassed to ask for help, ${ }^{20-22}$ an ED visit can play an important role in early identification and prevention of hazardous and harmful alcohol consumption. $^{23}$

Screening, Brief Intervention, and Referral to Treatment (SBIRT) is recommended for healthcare provider use in the $\mathrm{ED}$ to address alcohol related-concerns. ${ }^{24}{ }^{25}$ SBIRT begins with screening to identify whether current alcohol use is harmful and/or hazardous. Based on the screening result, healthcare provider interventions range from a brief intervention for patients meeting screening criteria for alcohol problems to a referral to specialised services for patients with a substance use disorder or alcohol dependence. ${ }^{26}$ Use of SBIRT in the ED with adolescents, however, is limited by ED clinicians' beliefs that alcohol-related visits require more time and resources than they can offer during care and limited training and support. ${ }^{27}{ }^{28}$ Computer-based SBIRT has emerged as a promising strategy to address implementation barriers in the ED. ${ }^{29-31}$ Recent efficacy trials of computer-based brief interventions have also reported reduced alcohol-related consequences among adolescent ED patients who have reported risky alcohol use, or past year alcohol use and aggression. $^{32} 33$

We conducted a pilot randomised controlled trial (RCT) to determine the feasibility and acceptability of a computer-based SBIRT intervention in the ED for adolescents with alcohol-related presentations. Our objectives were to: (1) estimate recruitment and retention rates, (2) assess perception of group allocation among ED and research staff, (3) evaluate the acceptability and feasibility of the intervention among adolescents, (4) obtain data on differences in alcohol consumption at different timepoints to estimate a sample required for a definitive RCT, and (5) examine changes in health and social services utilisation for alcohol use problems.

\section{METHODS}

The study design was a two-arm pilot RCT (standard ED care plus computer-based SBIRT versus standard ED care plus a computer-based sham) conducted in three pediatric EDs across Canada: the Stollery Children's Hospital (Edmonton, Alberta); IWK Health Centre (Halifax, Nova Scotia); and Alberta Children's Hospital (Calgary, Alberta). The Research Ethics Boards at each site approved the study.

\section{Eligibility and recruitment}

Between August 1, 2010 and February 28, 2013, research assistants (RAs) at each study site identified patients who presented to the ED with an alcohol-related chief complaint using an ED computer tracking system that indicated the reason for each patient's visit, or by direct referral from ED staff (nurses or attending physicians) that identified the visit was alcohol-related. RA schedules for recruitment were site specific; generally, peak hours for alcohol-related presentations were targeted (eg, between $1 \mathrm{pm}$ and $11 \mathrm{pm}$, Friday to Monday). Adolescents aged 12 to 17 years were eligible for the study if they reported drinking alcohol prior to ED visit and/ or had a positive laboratory result for alcohol in their medical record (ie, positive Blood Alcohol Content; $\mathrm{BAC}$ ).

Adolescents were ineligible for study participation if they: (1) required hospital admission (ie, were medically or psychiatrically unstable), (2) were unable to speak or understand English, (3) were currently enrolled in a treatment programme for alcohol use, (4) did not have the capacity to give informed consent as determined by their attending physician (eg, ongoing intoxication, violent), or (5) did not have regular access to a telephone (necessary for outcome measurement after the ED visit).

Adolescents who were not accompanied by a parent/ guardian, but who were considered by attending ED physicians to be mature minors (ie, mature enough to consent to their medical care), were eligible for enrolment if they otherwise met study eligibility requirements. Adolescents who were not considered mature minors were eligible for enrolment if they assented to study participation and had a consenting parent or guardian. Adolescents who were accompanied by a non-guardianship adult (eg, group home worker) but were not considered mature minors were unable to participate in the study.

We originally intended to exclude adolescents who reported other drug use and within the 24 hours prior to ED presentation (eg, cannabis), but removed this exclusion criterion 1 month after study start when we identified a concern from RAs that adolescents were reluctant to disclose other drug use during the screening process for the research study, and declined further screening to determine study eligibility. Removal of this criterion reduced the concern that we were not able to accurately identify or exclude those youth who had used other substances just prior to ED visit. We also felt that this change would not compromise the study's protocol or objectives. At the time of this protocol change, nine adolescents had been deemed ineligible for study enrolment due to report of other drug use or declining to disclose. 
Eligible adolescents deemed capable of providing informed consent/assent by the attending physician (ie, no longer intoxicated), and (if present) a parent/ guardian were approached by the RA in their assigned, private ED room to determine interest in study participation and confirm eligibility. The RA obtained informed consent from mature minor adolescents or a legal guardian with assent from the adolescent. Accompanying legal guardians were asked to provide privacy by leaving the ED room during actual study participation, after consent had been obtained.

\section{Randomisation}

Randomisation was performed after consent/assent and baseline data had been collected. Participants were assigned in a 1 to 1 allocation to either experimental or control group using a computer-generated randomisation schedule activated by logging into the study website. There was no stratification or planned restriction (eg, permuted blocks).

\section{Blinding}

Researchers and participants were blinded to allocation. Participants were informed the study was designed to provide an intervention for lifestyle behaviours. Attending physicians, nurses, and RAs were blinded to intervention assignment to reduce impact on usual clinical treatment.

\section{Experimental group: Computer-based SBIRT}

To reduce impact on usual clinical treatment, the computer-based SBIRT intervention was delivered to adolescents after medical examinations/care associated with the ED visit were complete. The intervention was a customised version of the Check Your Drinking tool (CYD; www.checkyourdrinking.net). The CYD tool has been previously evaluated in several RCTs involving young adult and adult drinkers. ${ }^{34-37}$ We used an iPad to deliver the intervention to facilitate ease of use at the adolescent's bedside; each iPad used a data plan for Internet access. The Alcohol Use Disorders Identification Test (AUDIT; 10 items), ${ }^{38}$ was embedded into the CYD tool to assess drinking patterns, alcohol dependence, and alcohol-related consequences.

The CYD tool used the adolescent's self-reported drinking patterns to generate personalised assessment feedback, a type of brief intervention based on normative feedback. ${ }^{39}$ Personalised assessment feedback included: (i) Gender- and age-specific referents: A summary of the adolescent's weekly alcohol consumption was compared with gender- and age-matched referents. ${ }^{40}$ Age referents were only available for ages 15-17 years, so 14 year old participants were provided with an explanation that their behaviours had been compared with older adolescents. (ii) Impact of alcohol consumption: Based on reported quantity and frequency of drinking, estimated financial costs of this consumption and caloric intake were calculated. A dose-response chart was also generated to highlight the likelihood of alcohol-related consequences, and a summary of adolescent reported consequences related to alcohol in the past year were presented as well as a general summary on the risks associated with alcohol use (eg, accidents, exposure to violence). (iii) Referral to resources: The intervention concluded with the provision of locally relevant, youth oriented mental health/addiction resources for problematic drinking (ie, for patients interested in specialty treatment services). Adolescents had the option to e-mail themselves a copy of the intervention's feedback. The time required to complete the intervention was $3-5$ min (2 $\min$ to administer $+1-3$ min to review feedback).

\section{Control group: Computer-based sham}

The computer-based sham was also delivered post-medical care. The sham was similar in format and duration as the computer-based SBIRT, but instead, engaged adolescents in a 10-item 'eating and exercise trivia' quiz from Kidzworld Media (kidzworld.com). Permission to deliver the quiz was obtained from Kidzworld Media prior to study start. Following each trivia question, a screen showed adolescents whether they answered the question correctly or incorrectly, and provided the correct response to each question with information explaining each response.

\section{Study procedures}

Following study enrolment, the RA collected baseline data and contact information using a paper-based form. The RA then logged into the study's secure website; this login activated the randomisation schedule. There was a several second delay to allow the RA to hand the study iPad to the adolescent to begin the intervention. This delay also served to blind the RA to intervention allocation. Adolescents were left alone in their ED room during the intervention, and were reassured that answers would not be disclosed to their ED care providers or parents/legal guardians accompanying them. The RA collected post-intervention data over the phone at 1- and 3 months. Adolescents received a $\$ 10$ gift card for participation in the study regardless of study completion.

\section{Data collection}

Study Recruitment and Retention

A study log was used by the RAs to track the number of adolescents who were screened, eligible and enrolled/ not enrolled in the study, and completed post-intervention measures at 1 - and 3 months.

\section{Demographic and ED Visit Characteristics}

Demographic data (age, sex), date of the ED visit, BAC (if available), and information on whether other substances had been used prior to ED presentation were collected by the RA from the adolescent's medical record. Contact information (telephone contact information, alternate telephone number, best time to call, e-mail) was collected verbally by the RA from the adolescent following study enrolment. 


\section{Perception of Group Allocation}

Perception of group allocation was assessed to determine whether there was a risk for contamination bias in a future study, and to determine whether there was a need to re-design study procedures to blind RA and ED staff to allocation. The RA, attending physician, and nurse completed a 1-item survey to indicate whether they thought the adolescent had been randomised to the experimental or control group. Response options were 'intervention group' (experimental group), 'don't know' or 'control group'. The survey item was filled out after the adolescent was discharged from the ED, which allowed for RAs and ED staff to account for post-intervention interactions that could have revealed allocation.

\section{Computer-based SBIRT Acceptability and Feasibility}

Computer-based SBIRT intervention acceptability and feasibility was collected post-intervention from adolescents allocated to the experimental group. Five items, designed specifically for this study, were administered to assess acceptability (satisfaction with the intervention, perceptions of the helpfulness, credibility of the personalised assessment feedback) and feasibility (time to completion, user friendliness) (Supplementary file). The research team reviewed the survey for face and content validity.

\section{Alcohol Consumption}

Data on alcohol consumption were collected at baseline and each post-intervention time-point. RAs verbally administered the consumption subscale of the Alcohol Use Disorders Identification Test (AUDIT-C), ${ }^{38}$ which consists of 3 items regarding the frequency and amount of alcohol consumption, and frequency of binge drinking (defined as six or more drinks on one occasion). The subscale score ranges from 0 to 12 .

\section{Health and Social Services Utilisation for Alcohol Use Problems}

Adolescents completed the Child and Adolescent Services Assessment (CASA) at baseline and each post-intervention time-point. The CASA is a valid and reliable self-report instrument designed to assess the use of community- and hospital-based health and social services; we focused each question so that we collected service use for an alcohol use problem. ${ }^{41}$ Adolescents were also asked two questions on receptivity to receiving services and eight questions on perceived barriers to services (Supplementary file).

\section{Outcomes}

The main outcomes were change in AUDIT-C scores from baseline to 1- and 3 months post-intervention. Secondary outcomes were study recruitment and retention rates, intervention acceptability and feasibility, perception of group allocation among ED and research staff, and change in health and social services utilisation from 30 days prior to the adolescent's ED visit (baseline) to 1-and 3 months post-intervention.

\section{Sample size}

We originally set out to determine the sample size necessary for a definitive RCT using primary outcome data from the pilot RCT. ${ }^{42}$ We estimated that we would need 20 subjects per group (40 total) at the 3 month post-intervention time-point to estimate standard deviations (SDs) and provide $80 \%$ confidence intervals (CIs) for SDs and 95\% CIs for recruitment and retention proportions with sufficient levels of precision in our calculations. The sample size for the pilot trial reflects this estimation.

\section{Analysis}

Descriptive statistics were used to summarise demographic and ED visit characteristics, recruitment and retention rates, healthcare utilisation, and intervention acceptability. Participant data was considered 'unknown/no response' if no data were recorded, data were missing, or answers were incomplete (ie, not decipherable).

The study's recruitment rate (the number of adolescents enrolled during the study period divided by the number of adolescents eligible to participate during the study period) was calculated to determine an overall timeline for a future trial and to assess the adequacy of recruitment at participating ED sites. Study retention rates (the number of adolescents who completed measures at each post-intervention time point) were calculated for 1- and 3 month follow-up time-points to assess the feasibility of a follow-up period in a definitive RCT.

Separate tables were produced to compare the adolescents' allocation to the perceptions of the RA, attending physician, and nurse. Fisher's exact test was used to assess the association between true and perceived allocation.

Alcohol consumption change scores were expressed as means with SDs. We carried the last value forward for participants that did not complete the primary outcome measure at post-intervention time-points. As the AUDIT-C has good sensitivity and specificity in identifying harmful and hazardous alcohol use among adolescent ED patients, ${ }^{43}{ }^{44}$ we also used the recommended cut-off score of 3 to identify harmful and hazardous drinking among participants using baseline AUDIT-C scores. ${ }^{43}$ SAS and Splus were used for all analyses.

\section{Results}

Recruitment and retention rates

Three hundred and forty adolescent ED patients were screened for study eligibility. One hundred and seventeen adolescents were eligible for study participation, and of these adolescents, 44 consented and were enrolled in the study ( $37.6 \%$ recruitment rate). The flow of participants through the trial is described in figure 1. Eighteen adolescents were allocated to the experimental group and received computer-based SBIRT. Twenty-six adolescents were allocated to the control group and received the computer-based sham. 


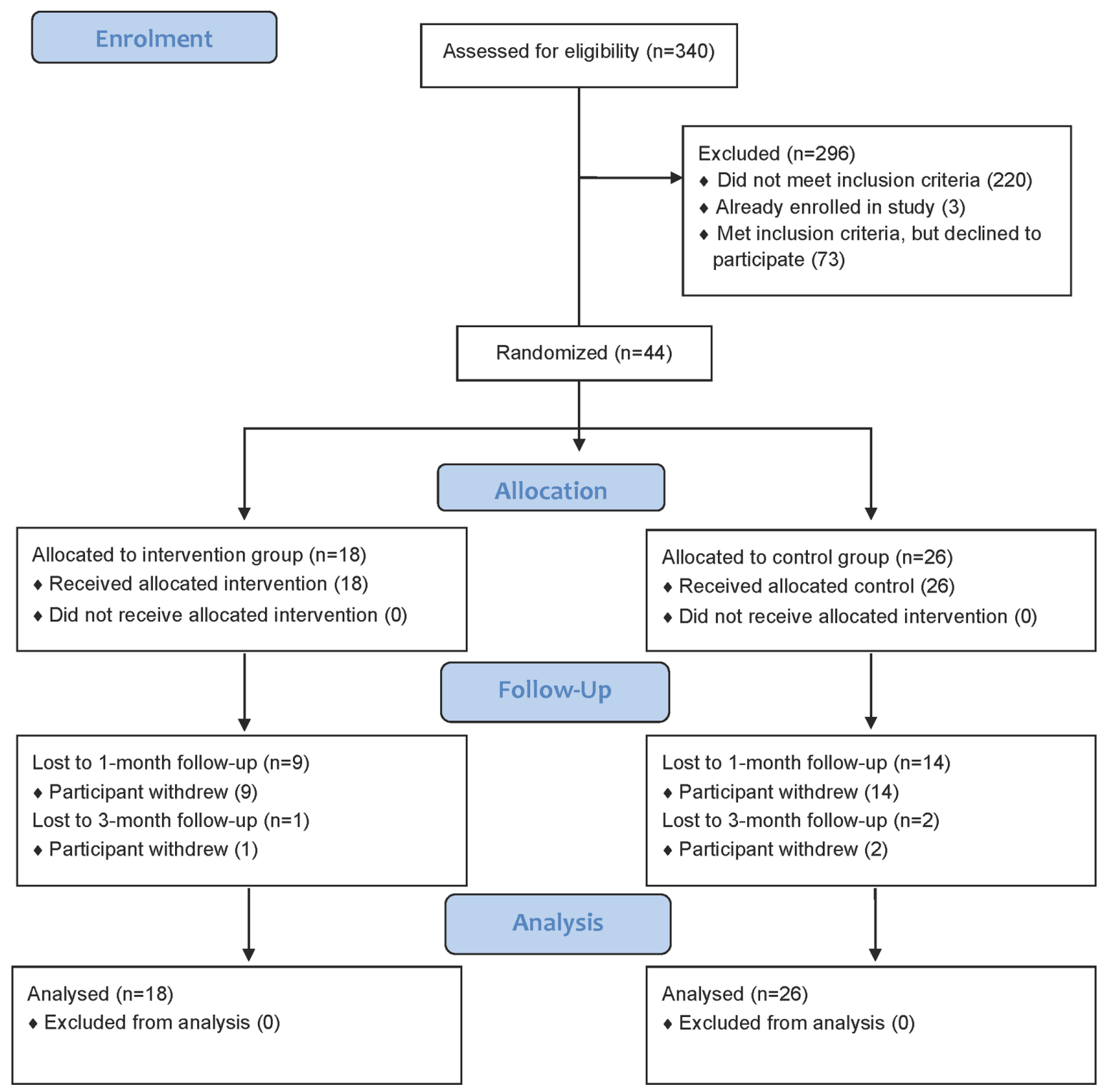

Figure 1 CONSORT diagram describing flow of participants through the study.

The imbalance between the intervention and control group allocation was due to the research team accessing the research website during the trial (January 2012) to generate intervention content for a media-related event. Each time a team member accessed the website he/she was randomly allocated to one of the two groups. Team members continued to access the website until they could generate content; thus, the randomisation sequence was disrupted as participants were enrolled. Because no interim analyses were planned, the imbalance went undetected during study conduct. The study's overall retention rate at 1 month post-intervention was $47.7 \%(21 / 44)$ and $40.9 \%(18 / 44)$ at 3 months post-intervention. No adolescents allocated to either group were excluded from the analyses.

\section{Description of the study sample}

Characteristics of participants are shown in table 1. The average age of participants was 14.89 years (SD 0.97) and the majority of participants were female $(31 / 44)$. Seventy-five percent of participants presented to the ED for emergencies related solely to alcohol use (33.44). At baseline, $88.6 \%$ of participants in the study had an AUDIT-C score $\geq 3$ indicating harmful and hazardous alcohol consumption.

\section{Perception of group allocation}

table 2 provides the raw counts and percentages of correct/incorrect guesses of allocations by research staff, nurses, and physicians. There was no evidence of a statistically significant relationship between which interventions adolescents were allocated to and which interventions ED staff (nurses, $\mathrm{p}=0.26$; physicians, $\mathrm{p}=1.00$ ) and RAs $(\mathrm{p}=0.63)$ thought they received.

\section{Computer-based SBIRT acceptability}

Of the 18 adolescents allocated to the intervention, 14 $(77.8 \%)$ provided feedback on the computer-based 
Table 1 Baseline characteristics of enrolled adolescents with alcohol-related ED visits

Experimental group $(n=18)$ Control group $(n=26)$ All $(n=44)$

\begin{tabular}{|c|c|c|c|}
\hline \multicolumn{4}{|l|}{ Age, n (\%) } \\
\hline 13 years & $1(5.6 \%)$ & $2(7.7 \%)$ & $3(6.8)$ \\
\hline 14 years & $3(16.7 \%)$ & $9(34.6 \%)$ & $12(27.3)$ \\
\hline 16 years & $6(33.3 \%)$ & $6(23.1 \%)$ & $12(27.3)$ \\
\hline 17 years & $1(5.6 \%)$ & $0(0 \%)$ & $1(2.3)$ \\
\hline Male & $6(33.3 \%)$ & $7(26.9 \%)$ & $13(29.5)$ \\
\hline Female & $12(66.7 \%)$ & $19(73.1 \%)$ & $31(70.5)$ \\
\hline \multicolumn{4}{|l|}{ AUDIT-C, n (\%) } \\
\hline Met cut-score of 3 for harmful and hazardous drinking & $15(83.3 \%)$ & $24(92.3 \%)$ & $39(88.6)$ \\
\hline \multicolumn{4}{|l|}{ Report of using other substances prior to ED visit, $n(\%)$} \\
\hline Marijuana and ecstasy & $1(5.6 \%)$ & $0(0 \%)$ & $1(2.3)$ \\
\hline None & $14(77.8 \%)$ & $19(73.1 \%)$ & $33(75.0)$ \\
\hline
\end{tabular}

SBIRT intervention (table 3). The majority were satisfied with the computer-based SBIRT intervention: $55.6 \%$ felt it to be informative regarding drinking behaviours and its effects (strongly agreed/agreed) while $22.3 \% \mathrm{did}$ not (disagreed/strongly disagreed), and $61.1 \%$ felt they could quickly finish the intervention while $16.7 \%$ felt they could not. Adolescents were divided on the credibility of normative feedback provided as part of the intervention: $44.4 \%$ agreed/strongly agreed that it was believable, $16.7 \%$ disagreed, and $16.7 \%$ were unsure if the feedback was believable or not; $22.2 \%$ of adolescents did not respond.

\section{Alcohol consumption change scores and sample size for a definitive RCT}

We do not present alcohol consumption change scores, as we did not use these scores to determine a sample size for the definitive RCT. This action was based on decision made after the conclusion of the pilot RCT, which was to change the primary outcome from alcohol consumption to alcohol-related consequences for the definitive trial. When designing the pilot trial, we used extant literature ${ }^{45}$ to select alcohol consumption as the primary outcome. However, recent consultations with ED clinicians representing potential recruitment sites for the definitive trial have revealed that clinicians are most interested in the effect that computer-based SBIRT has on alcohol-related consequences since these consequences are often what bring adolescents to the ED for care. Alcohol consumption change scores are reported in the clinical trials record (www.clinicaltrials.gov; NCT01146665).

\section{Health and social services Utilisation}

Forty-two adolescents completed the CASA instrument at baseline $(95.5 \%), 21$ completed the measure at 1 month post-intervention (47.7\%), and 18 completed it at 3 month post-intervention $(40.9 \%)$. We did not formally assess for differences

Table 2 Intervention allocation status and perceived allocation

\begin{tabular}{|c|c|c|c|c|}
\hline & \multicolumn{3}{|c|}{ Allocation Guess, n (row \%) } & \multirow[b]{2}{*}{ Total } \\
\hline & Intervention & Control & Do not know & \\
\hline \multicolumn{5}{|c|}{ Adolescents allocated to intervention } \\
\hline ED Nurses & 4 & 6 & 8 & 18 \\
\hline ED Physicians & 5 & 0 & 13 & 18 \\
\hline \multicolumn{5}{|c|}{ Adolescents allocated to control } \\
\hline Research Staff & 16 & 9 & 1 & 26 \\
\hline ED Nurses & 9 & 3 & 14 & 26 \\
\hline ED Physicians & 7 & 1 & 18 & 26 \\
\hline
\end{tabular}


Table 3 Adolescent feedback on the computer-based SBIRT intervention, $\mathrm{n}(\%)$

\begin{tabular}{ll}
\hline Liked the way the intervention looked & \\
\hline Strongly agreed & $1(5.6 \%)$ \\
\hline Agreed & $11(61.1 \%)$ \\
Unsure & $1(5.6 \%)$ \\
Disagreed & $1(5.6 \%)$ \\
Strongly disagreed & $0(0 \%)$ \\
Unknown/No response & $4(22.2 \%)$ \\
Felt the intervention was easy to use & \\
Strongly agreed & $2(11.1 \%)$ \\
Agreed & $9(50.0 \%)$ \\
Unsure & $1(5.6 \%)$ \\
Disagreed & $2(11.1 \%)$ \\
Strongly disagreed & $0(0 \%)$ \\
Unknown/No response & $4(22.2 \%)$ \\
\hline
\end{tabular}

Felt they could quickly finish the intervention

\begin{tabular}{ll} 
Strongly agreed & $3(16.7 \%)$ \\
Agreed & $8(44.4 \%)$ \\
Unsure & $0(0 \%)$ \\
Disagreed & $3(16.7 \%)$ \\
Strongly disagreed & $0(0 \%)$ \\
Unknown/No response & $4(22.2 \%)$ \\
Felt the intervention helped them think about drinking \\
behaviours and effects \\
Strongly agreed \\
Agreed & $1(5.6 \%)$ \\
Unsure & $9(50.0 \%)$ \\
Disagreed & $0(0 \%)$ \\
Strongly disagreed & $3(16.7 \%)$ \\
Unknown/No response & $1(5.6 \%)$ \\
\hline
\end{tabular}

Felt the feedback the intervention gave was believable

\begin{tabular}{ll} 
Strongly agreed & $2(11.1 \%)$ \\
Agreed & $6(33.3 \%)$ \\
Unsure & $3(16.7 \%)$ \\
Disagreed & $3(16.7 \%)$ \\
Strongly disagreed & $0(0 \%)$ \\
Unknown/No response & $4(22.2 \%)$ \\
\hline
\end{tabular}

in health and social services utilisation during the study due to the large amount of missing data. In table 4, we present the most complete data set for this outcome, which was information collected at baseline, the time of the ED visit. At this time-point, $50 \%$ of adolescents reported accessing health and social services for an alcohol use problem $(22 / 44)$, 18 reported never utilising services $(40.9 \%)$; data were missing for four adolescents $(9.1 \%)$. The most common resources for help reported were seeing a psychiatrist, psychologist or social worker; help from family and friends; and school-based services (table 4).

Forty adolescents answered treatment receptivity questions during the ED visit (missing data, 9.1\%). When asked whether it was a 'good or definitely good idea' or 'bad or definitely idea' for someone to seek help for an alcohol use problem, $79.5 \%$ of adolescents responded favourably. When asked about the helpfulness of treatment from a doctor or counsellor for an alcohol use problem, 56.8\% of adolescents replied felt they can 'help/definitely can help', while $15.9 \%$ felt they 'cannot help/definitely cannot help'; $18.2 \%$ were unsure as to whether a physicians or counsellor could help.

Questions related to perceived barriers for health and social services use for alcohol use problems were answered by $25-27$ adolescents during the ED visit (missing data range, $38.6 \%-43.2 \%$ ). The majority of adolescents who responded did not have concerns regarding barriers, but percentage of adolescents did, and for some, these barriers affected their service use (table 5).

\section{DISCUSSION}

This RCT piloted procedures and obtained data on intervention acceptability to determine the feasibility of a definitive RCT of the effectiveness of a computer-based brief intervention in the ED. Three key lessons were learnt from conducting this pilot study: (1) The computer-based SBIRT intervention was acceptable to adolescents and feasible to deliver in the ED, but adjustments to the intervention are needed to improve the credibility of the personalised assessment feedback in the intervention. (2) A definitive RCT to test the effectiveness of the intervention is feasible if protocol adjustments are made to improve recruitment and retention rates, and increase the number of study sites and research staff. (3) The primary outcome for the definitive trial should be alcohol-related consequences so that the trial is measuring the outcome considered by ED clinicians to be most clinically important for SBIRT delivery.

Providing computer-based SBIRT is a promising strategy to address clinicians' concerns that they have limited time, resources, support and training to address alcohol-related concerns during the ED visit. ${ }^{27} 28$ Computer-based SBIRT may also standardise care so that ineffective approaches (eg, cautionary appeals) are avoided. The fact that $50 \%$ of adolescents in this pilot study reported a prior history of accessing services for alcohol use reinforces the need for a more comprehensive approach to the management of youth who present to the ED with an alcohol-related chief complaint. Results suggest that adolescents found SBIRT to be informative, and that computer-delivery required little time. However, because adolescents were divided on the credibility of the normative feedback provided by the intervention (the brief intervention component of SBIRT), the approach we took for intervention delivery requires reconsideration. In this pilot study, we adopted 
Table 4 Type of health and social services used for alcohol use problems reported at the time of the ED visit.

\begin{tabular}{lll}
\hline \multirow{2}{*}{ Service } & \multicolumn{1}{c}{$\mathbf{n}(\%)$} \\
\cline { 2 - 3 } & Used (ever) & Used in the last 3 months \\
\hline Hospital & $3(6.8 \%)$ & $0(0 \%)$ \\
Psychiatrist/Psychologist/Social Worker & $6(13.6 \%)$ & $5(11.4 \%)$ \\
Clinic & $4(9.1 \%)$ & $1(2.3 \%)$ \\
Crisis Services & $3(6.8 \%)$ & $1(2.3 \%)$ \\
General Physician & $7(15.9 \%)$ & $2(4.5 \%)$ \\
School-based Services & $5(11.4 \%)$ & $4(9.1 \%)$ \\
\hline Family/Friends & $5(11.4 \%)$ & $8(18.2 \%)$ \\
Hotline & $1(2.3 \%)$ & $1(2.3 \%)$ \\
Self-help & $0(0 \%)$ & $1(2.3 \%)$ \\
Internet & $0(0 \%)$ & $1(2.3 \%)$ \\
Missing data & $2(4.5 \%)$ & $2(4.5 \%)$ \\
\hline
\end{tabular}

a self-led approach; that is, adolescents undertook SBIRT without interacting with attending physicians and/or nurses. For the planned definitive RCT, we propose that ED clinicians be involved in the delivery of computer-based SBIRT. Whether it is physician, nursing or specialised mental health staff, clinician involvement would provide an opportunity for adolescents to discuss the normative feedback and for clinicians to address any questions or concerns about the feedback. These discussions, in turn, may reinforce the credibility of the feedback. That some adolescents in this study delayed or did not seek services because of negative feelings and/or the sensitivity of the topic necessitates that clinicians be aware of these barriers to healthcare seeking when interacting with adolescents.

With the experimental group of the definitive trial proposed as 'ED clinician delivery of computer-based SBIRT during routine care', we believe that the most appropriate comparator is 'ED clinician delivery of routine ED care' rather than a sham intervention. The sham intervention was more appropriate when computer-based SBIRT was self-led by adolescents. Thus, while this pilot study has indicated that the measures we put into place to reduce the possibility of unblindingcomputer-based randomisation with the randomisation scheme prepared by a biostatistician and the use of a sham intervention-were effective, the definitive trial will be conducted as open-label. ED clinicians will not be blinded to allocation, as they will deliver the interventions. Enrolled adolescents will be informed that they will be taking part in a study comparing different types of alcohol care and will not be made aware of our hypothesis; however, they will be the primary outcome assessors as outcomes will be self-reported.

We also believe that the definitive trial is best conducted as a multi-site, cluster RCT. The pilot trial recruitment rate and the number of adolescents who declined trial participation $(25 \%)$ indicate that increasing the number of participating EDs and RA staffing are critical to the success of a definitive trial. Due to budgetary constraints for the pilot RCT, we recruited adolescents from three pediatric EDs; however, there are 14 pediatric EDs across Canada in the Pediatric Emergency Research Canada (PERC) network. We have confirmed participation of 10 of the 14 EDs in the PERC network for the definitive trial. The trial will be designed as a cluster RCT because clinician delivery of computer-based SBIRT introduces a risk of contamination if we randomise at the patient level. In the definitive RCT, random assignment will be based on stratified clusters with month as the unit of randomisation and ED sites as strata. This approach reduces the risk of contamination bias that could be introduced by clinicians with patient level randomisation. Month is the appropriate unit of randomisation for this study as other potential choices (ED, week) will not allow us to also study SBIRT implementation in the ED sites over a sufficient time-period. Months will be randomised within strata in a 1:1 ratio to either experimental (ED clinician delivery of computer-based SBIRT during routine ED care) or control (ED clinician delivery of routine ED care) conditions. A calendar of the randomisation schedule for each ED will be provided to research staff and monitored for adherence. With randomisation now external to the website used for SBIRT delivery, we have also eliminated the potential to disrupt randomisation as we did in the pilot RCT.

With 10 EDs participating in the definitive trial, we have a much larger population to recruit from, which will address our concerns about the modest recruitment and retention rates in the pilot RCT. With respect to recruitment, in 2016, these EDs treated 976 adolescents with visits for alcohol-related concerns. Using baseline data from our pilot trial, we expect that $89 \%$ of these adolescents will screen positive for harmful and hazardous alcohol use. We will aim to enrol these adolescents into the definitive trial. In the same year, these EDs also treated 853 mental health visits that involved harmful and hazardous alcohol use. Discussions with ED clinicians representing the recruitment sites identified that these adolescents are also a preferred 


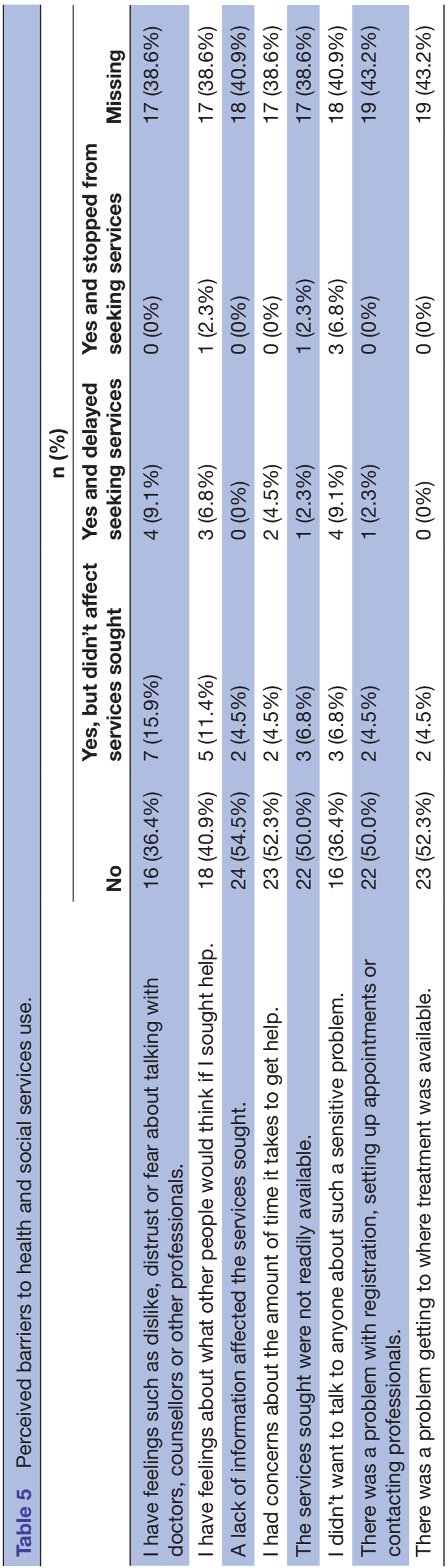

population for ED SBIRT delivery given the adolescents' underlying problematic alcohol use. Thus, we will also aim to enrol these adolescents into the definitive trial. Taking these annual presentation numbers together, there are 1722 adolescents potentially eligible each year for participation in the definitive trial ( 15 eligible patients each month per ED). The sample size calculation for the definitive trial will take this information into account. Regarding retention, that the 3 month retention rate in this pilot RCT $(40.9 \%)$ was lower than what was reported in a recent efficacy trial of computer-based SBIRT $(50.2 \%)$ conducted by Cunningham et al, ${ }^{32}$ suggests that strategies to mitigate study attrition are important for our team to consider for the definitive trial. Adolescents in the Cunningham trial received remuneration of $\$ 35$ at the 3 month follow-up time-point; use of Web links email to collect follow-up data (versus telephone follow-up) was also used..$^{32}$ We will use these strategies in the definitive trial to increase study retention at follow-up.

Finally, our decision to define alcohol-related consequences as the primary outcome for the definitive trial based on ED clinician feedback is in keeping with two trials published since we designed our pilot trial, that also defined this outcome as a primary outcome of interest. ${ }^{32} 33$ In the definitive trial, we will measure alcohol-related consequences 3 months after intervention delivery as this end-point reflects a clinically important time period (eg, a sustained effect of a brief intervention).

Of note, two additional aspects raised during the conduct of this study also need to be addressed during the design of the definitive trial. Our change in protocol to remove the exclusion criterion related to other drug use 1 month after study start was not expected. We did not anticipate adolescents' hesitation to disclose this use in the context of a research study. Of the nine participants who were excluded prior to the protocol change, we do not know how many were eligible for study participation as they did not answer the question on other drug use or questions that followed related to study eligibility. We also do not know of those who were eligible, how many would have agreed to participate in the study. Because we plan to study the effectiveness of the computer-based intervention in the definitive trial, we will not include this exclusion criterion in the definitive trial. A sub-group analysis will be conducted to determine whether adolescents who report poly-substance use at the time of the ED visit have different outcomes than adolescents who report only alcohol use. Second, the high percentage of missing healthcare utilisation data using the CASA suggests that an instrument with fewer items (the CASA asks $>100$ questions) is needed if we are to study this outcome in a definitive trial. While trials of computer-based SBIRT have not examined healthcare utilisation, ${ }^{32} 33$ other measures do exist. ${ }^{46}$ 


\section{CONCLUSION}

The results from this pilot RCT indicate that a definitive RCT to assess the effectiveness of computer-based SBIRT in an adolescent population may be feasible in a Canadian context. Adolescents found SBIRT to be informative and that computer-delivery required little time. Adjustments to SBIRT delivery are necessary, however, to address concerns about intervention credibility. Increasing the number of recruiting pediatric EDs, putting measures into place to reduce study attrition at outcome measurement time-points, and re-examining outcome measures to reduce participant burden are also important to consider in the design of a future trial.

\section{Author affiliations}

${ }^{1}$ Department of Pediatrics, University of Alberta, Edmonton, Canada

${ }^{2}$ Women and Children's Health Research Institute, Edmonton, Canada

${ }^{3}$ Faculty of Nursing, University of Alberta, Edmonton, Canada

${ }^{4}$ Department of Emergency Medicine, University of Alberta, Edmonton, Canada

${ }^{5}$ Department of Emergency Medicine, Dalhousie University, Halifax, Canada

${ }^{6}$ School of Public Health, University of Alberta, Edmonton, Canada

${ }^{7}$ Departments of Pediatrics and Physiology and Pharmacology, Cummings School of Medicine, University of Calgary, Calgary, Canada

${ }^{8}$ School of Epidemiology, Public Health \& Preventive Medicine, University of Ottawa, Ottawa, Canada

\section{Acknowledgements This study was conducted with the Pediatric Emergency} Research Canada (PERC) network.

Contributors Dr Newton conceptualised and designed the study, obtained funding for the study and supervised the study, acquired the data, analysed and interpreted the data, helped draft the initial manuscript, critically revised the manuscript for important intellectual content, and approved the final manuscript as submitted. Ms Dow contributed to the design of the study, acquired the data, analysed and interpreted the data, drafted the initial manuscript, critically revised the manuscript for important intellectual content, and approved the final manuscript as submitted. Dr Dong contributed to the design of the study, obtained funding for the study and supervised the study, analysed and interpreted the data, critically revised the manuscript for important intellectual content, and approved the final manuscript as submitted. Dr Rosychuk contributed to the design of the study, oversaw data analysis, critically revised the manuscript for important intellectual content, and approved the final manuscript as submitted. Dr Wild contributed to the design of the study, interpreted the data, critically revised the manuscript for important intellectual content, and approved the final manuscript as submitted. Ms Fitzpatrick supervised the study, interpreted the data, critically revised the manuscript for important intellectual content, and approved the final manuscript as submitted. Dr Johnson contributed to the design of the study, interpreted the data, critically revised the manuscript for important intellectual content, and approved the final manuscript as submitted. Dr Ali contributed to the design of the study, interpreted the data, critically revised the manuscript for important intellectual content, and approved the final manuscript as submitted. Dr Colman contributed to the design of the study, interpreted the data, critically revised the manuscript for important intellectual content, and approved the final manuscript as submitted.

Funding Funding for this project was provided by a grant from the Women and Children's Health Research Institute (Edmonton, AB) and the Norlien Foundation (now the Palix Foundation; Calgary, AB). Dr. Newton holds a ClHR New Investigator Award. During this work, Dr. Rosychuk held a Health Scholar award from Alberta Innovates - Health Solutions. Dr. Colman holds a Tier II Canada Research Chair. The authors have no other competing interests to declare.

Competing interests None declared.

Patient consent Data are anonymised and grouped. We present no identifiable medical information.

Ethics approval University of Alberta Health Research Ethics Board, University of Calgary Health Research Ethics Board, IWK Health Centre Research Ethics Board.

Provenance and peer review Not commissioned; externally peer reviewed.
Data sharing statement Additional data for the primary outcome are available to researchers, and can be found in the clinicaltrials.gov record (NCT01146665). This information is noted in the manuscript.

Open Access This is an Open Access article distributed in accordance with the Creative Commons Attribution Non Commercial (CC BY-NC 4.0) license, which permits others to distribute, remix, adapt, build upon this work non-commercially, and license their derivative works on different terms, provided the original work is properly cited and the use is non-commercial. See: http://creativecommons.org/ licenses/by-nc/4.0/

(C) Article author(s) (or their employer(s) unless otherwise stated in the text of the article) 2017. All rights reserved. No commercial use is permitted unless otherwise expressly granted.

\section{REFERENCES}

1. Masten AS, Faden VB, Zucker RA, et al. Underage drinking: a developmental framework. Pediatrics 2008;121 Suppl 4(suppl 4):S235-S251.

2. Grant BF, Dawson DA. Age at onset of alcohol use and its association with DSM-IV alcohol abuse and dependence: results from the National Longitudinal Alcohol Epidemiologic Survey. J Subst Abuse 1997;9:103-10.

3. Bonomo YA, Bowes G, Coffey C, et al. Teenage drinking and the onset of alcohol dependence: a cohort study over seven years. Addiction 2004;99:1520-8.

4. Viner RM, Taylor B. Adult outcomes of binge drinking in adolescence: findings from a UK national birth cohort. J Epidemiol Community Health 2007;61:902-7.

5. D'Amico EJ, Ellickson PL, Collins RL, et al. Processes linking adolescent problems to substance-use problems in late young adulthood. J Stud Alcohol 2005;66:766-75.

6. Kessler RC, Berglund P, Demler O, et al. Lifetime prevalence and age-of-onset distributions of DSM-IV disorders in the National Comorbidity Survey replication. Arch Gen Psychiatry 2005;62:593-602.

7. DeWit DJ, Adlaf EM, Offord DR, et al. Age at first alcohol use: a risk factor for the development of alcohol disorders. Am J Psychiatry 2000;157:745-50.

8. Rohde P, Lewinsohn PM, Seeley JR, et al. Psychosocial functioning of adults who experienced substance use disorders as adolescents. Psychol Addict Behav 2007;21:155-64.

9. Conway KP, Swendsen J, Husky MM, et al. Association of Lifetime Mental Disorders and subsequent alcohol and illicit drug use: results from the National Comorbidity Survey-Adolescent Supplement. J Am Acad Child Adolesc Psychiatry 2016;55:280-8.

10. Grant BF, Stinson FS, Harford TC. Age at onset of alcohol use and DSM-IV alcohol abuse and dependence: a 12-year follow-up. $J$ Subst Abuse 2001;13:493-504.

11. Pitkänen T. Problem drinking and psychological well-being: a fiveyear follow-up study from adolescence to young adulthood. Scand J Psychol 1999;40:197-207.

12. Hingson RW, Heeren $T$, Jamanka $A$, et al. Age of drinking onset and unintentional injury involvement after drinking. JAMA 2000;284:1527-33

13. Hingson RW, Zha W. Age of drinking onset, alcohol use disorders, frequent heavy drinking, and unintentionally injuring oneself and others after drinking. Pediatrics 2009;123:1477-84.

14. Champion $\mathrm{H}$, Wagoner $\mathrm{K}$, Song $\mathrm{EY}$, et al. Adolescent date fighting victimization and perpetration from a multi-community sample: associations with substance use and other violent victimization and perpetration. Int J Adolesc Med Health 2008;20:419-29.

15. Clark DB, Martin CS, Cornelius JR. Adolescent-onset substance use disorders predict young adult mortality. J Adolesc Health 2008;42:637-9.

16. Bernstein E, Bernstein JA, Stein JB, et al. SBIRT in emergency care settings: are we ready to take it to scale? Acad Emerg Med 2009;16:1072-7.

17. Burke PJ, O'Sullivan J, Vaughan BL. Adolescent substance use: brief interventions by emergency care providers. Pediatr Emerg Care 2005;21:770-6.

18. Elder RW, Shults RA, Swahn MH, et al. Alcohol-related emergency department visits among people ages 13 to 25 years. J Stud Alcohol 2004;65:297-300.

19. Newton AS, Ali S, Johnson DW, et al. A 4-year review of pediatric mental health emergencies in Alberta. CJEM 2009;11:447-54.

20. D'amico EJ. Factors that impact adolescents' intentions to utilize alcohol-related prevention services. J Behav Health Serv Res 2005;32:332-40. 
21. Cunningham JA, Sobell LC, Sobell MB, et al. Barriers to treatment: why alcohol and drug abusers delay or never seek treatment. Addict Behav 1993;18:347-53.

22. Klein JD, McNulty M, Flatau CN. Adolescents' Access to Care. Arch Pediatr Adolesc Med 1998;152:676-82.

23. Academic ED SBIRT Research Collaborative. the impact of screening, brief intervention, and referral for treatment on emergency department patients' alcohol use. Ann Emerg Med 2007;50:699-710.

24. American College of Emergency Physicians.. Alcohol screening in the Emergency Department. www.acep.org/Clinical---PracticeManagement/Alcohol-Screening-in-the-Emergency-Department/. (Accessed October 30, 2015).

25. Higgins-Biddle J, Hungerford D, Cates-Wessel K. Screening and Brief Interventions (SBI) for Unhealthy Alcohol Use: A Step-By-Step Implementation Guide for Trauma Centers.. . Atlanta, GA: Centers for Disease Control and Prevention, National Center for Injury Prevention and Control, 2009:1-46.

26. American academy of Pediatrics Committee on Substance abuse. substance use screening, brief intervention, and referral to treatment. Pediatrics 2016;138. e20161210.

27. Mabood N, Ali S, Dong KA, et al. Experiences of pediatric emergency physicians in providing alcohol-related care to adolescents in the emergency department. Pediatr Emerg Care 2013;29:1260-5.

28. Chun TH, Spirito A, Rakowski W, et al. Beliefs and practices of pediatric emergency physicians and nurses regarding counseling alcohol-using adolescents: can counseling practice be predicted? Pediatr Emerg Care 2011;27:812-25.

29. Harris SK, Knight JR. Technology-based alcohol screening and brief interventions in medical settings. Alcohol Res 2014;36:63-79.

30. Ondersma SJ, Grekin ER, Svikis D. The potential for technology in brief interventions for substance use, and during-session prediction of computer-delivered brief intervention response. Subst Use Misuse 2011;46:77-86.

31. Harris SK, Louis-Jacques J, Knight JR. Screening and brief intervention for alcohol and other abuse. Adolesc Med State Art Rev 2014;25:126-56.

32. Cunningham RM, Chermack ST, Ehrlich PF, et al. Alcohol interventions among underage drinkers in the ED: a Randomized Controlled Trial. Pediatrics 2015;136:e783-e793.

33. Walton MA, Chermack ST, Shope JT, et al. Effects of a brief intervention for reducing violence and alcohol misuse among adolescents: a randomized controlled trial. JAMA 2010;304:527-35.

34. Cunningham JA. Comparison of two internet-based interventions for problem drinkers: randomized controlled trial. J Med Internet Res 2012;14:e107.
35. Cunningham JA, Wild TC, Cordingley J, et al. Twelve month followup results from a randomized controlled trial of a brief personalized feedback intervention for problem drinkers. Alcohol Alcohol 2010;45:247-51. 35 Doumas DM, McKinley LL, Book P. evaluation of two web-based alcohol interventions for mandated college students. J Subst Abuse 2009;36:65-74.

36. Cunningham JA, Wild TC, Cordingley J, et al. A randomized controlled trial of an internet-based intervention for alcohol abusers. Addiction 2009;104:2023-32.

37. Cunningham JA, Humphreys K, Kypri K, et al. Formative evaluation and three-month follow-up of an online personalized assessment feedback intervention for problem drinkers. J Med Internet Res 2006;8:e5.

38. Babor T, Higgins-Biddle J, Saunders J, et al. The alcohol use identification test: guidelines for primary caregivers. 2001. 2nd ed. Geneva.: World Health Organization, 2016. http://www.talkingalcohol. com/files/pdfs/WHO_audit.pdf.

39. Berkowitz A. An overview of the social norms approach. In: Lederman LC, Stewart LP, eds. Changing the culture of College Drinking: a socially Situated Health Communication Campaign. Cresskill, NJ: hampton Press, 2005. Chapter 13.

40. In: Adlaf EM, Demers A, Gliksman L, eds. Canadian Campus survey 2004. Toronto: Centre for Addiction and Mental Health, 2005.

41. Ascher BH, Z. Farmer EM, Burns BJ, et al. The child and Adolescent Services Assessment (CASA): Description and psychometrics. J Emot Behav Disord 1996;4:12-20.

42. Lancaster GA, Dodd S, Williamson PR. Design and analysis of pilot studies: recommendations for good practice. J Eval Clin Pract 2004;10:307-12.

43. Kelly TM, Donovan JE, Chung T, et al. Brief screens for detecting alcohol use disorder among 18-20 year old young adults in emergency departments: comparing AUDIT-C, CRAFFT, RAPS4QF, FAST, RUFT-Cut, and DSM-IV 2-Item Scale. Addict Behav 2009;34:668-74

44. Chung T, Colby SM, Barnett NP, et al. Alcohol use disorders identification test: factor structure in an adolescent emergency department sample. Alcohol Clin Exp Res 2002;26:223-31.

45. Maio RF, Shope JT, Blow FC, et al. A randomized controlled trial of an emergency department-based interactive computer program to prevent alcohol misuse among injured adolescents. Ann Emerg Med 2005;45:420-9.

46. Bhandari A, Wagner T. Self-reported utilization of health care services: improving measurement and accuracy. Med Care Res Rev 2006;63:217-35. 